Citation: S. Pinna (2020) Sui caratteri di spiccata marittimità dei climi di alta montagna. Bollettino della Società Geografica Italiana serie 14, 3(1): 51-57. doi: 10.36253/bsgi.v3i1.882

Copyright: @ 2020 S. Pinna. This is an open access, peer-reviewed article published by Firenze University Press (http://www.fupress.com/bsgi) and distributed under the terms of the Creative Commons Attribution License, which permits unrestricted use, distribution, and reproduction in any medium, provided the original author and source are credited.

Data Availability Statement: All relevant data are within the paper and its Supporting Information files.

Competing Interests: The Author(s) declare(s) no conflict of interest.

\section{Sui caratteri di spiccata marittimità dei climi di alta montagna}

\author{
Significant Maritime Characters of High Mountain Climates
}

\author{
Sergio Pinna \\ Dipartimento di Civiltà e forme del Sapere, Università di Pisa, Italia \\ E-mail: sergio.pinna@unipi.it
}

\begin{abstract}
The continentality level depends on several geographical factors, such as altitude which also plays an important role. In effect, the aim of this work is to carry out some simple tests related to the relationship continentality-altitude, by: a) calculating the regressions between altitude and a series of indices deriving from the analysis of the yearly march of temperature for each location examined; b) evaluating the characteristics of the thermal regime of some high mountain localities. The results obtained confirm a trend towards for a marked increase in the degree of oceanicity of the climate along with increasing altitude.
\end{abstract}

Keywords: Continentality, oceanicity, altitude, climate indexes.

Riassunto. Il livello di continentalità dipende da vari fattori geografici, fra i quali anche l'altitudine gioca un ruolo rilevante. In effetti, l'obiettivo del presente lavoro è stato proprio quello di condurre delle semplici verifiche in merito al rapporto continentalità-altitudine, sia calcolando alcune regressioni fra la quota sul mare ed una serie di indicatori derivanti dal quadro delle temperature medie mensili delle singole località censite, sia valutando le caratteristiche del regime termico di alcune località di alta montagna. I risultati ottenuti confermano la tendenza ad una marcata riduzione della continentalità con l'aumentare dell'altitudine.

Parole chiave: continentalità, marittimità, altitudine, indici climatici.

\section{Introduzione}

Per quanto concerne le caratteristiche termiche, la distinzione più evidente fra i climi continentali e quelli marittimi (detti anche oceanici) è costituita dalle escursioni, che nei primi tendono ad essere assai più ampie rispetto ai secondi. In effetti, l'indicatore fondamentale per ottenere delle classificazioni climatiche sotto tali aspetti è dato dall'escursione annua $\left(\mathrm{E}_{\mathrm{A}}\right)$, cioè dalla differenza fra la temperatura media del mese più caldo e quella del più freddo.

$\mathrm{La}_{\mathrm{A}}$ è però fortemente influenzata dalla latitudine, in ragione delle crescenti differenze stagionali nell'energia solare in arrivo al suolo, che si regi- 
strano procedendo dall'equatore in direzione dei poli; ne deriva perciò che, quando si vogliano effettuare comparazioni fra località poste a latitudini significativamente diverse, si deve necessariamente tenere conto di questo. Per fare dei semplici esempi, possiamo dire che Porto ha una $\mathrm{E}_{\mathrm{A}}$ di $10,6^{\circ}$ e che tale parametro a Salamanca, sempre nel bacino del Douro ma a $250 \mathrm{~km}$ dal mare, si attesta invece a $17,7^{\circ}$. Un valore pressoché identico a quest'ultimo lo ritroviamo però nei dati di Kristiansand, una città sulla costa meridionale della Norvegia, in questo caso posta ad una latitudine di 17 gradi maggiore rispetto alle due sopra citate località.

In tema di continentalità, la questione della "distanza dal mare" è comunque assai complessa, perché legata a fattori che possono agire su differenti scale geografiche. Il vero clima marittimo riguarda i fronti continentali direttamente esposti all'arrivo dei venti dall'oceano, mentre le regioni costiere situate sul versante opposto hanno livelli di continentalità assai più elevati, nonostante la loro prossimità con la massa marina; per apprezzare questo fatto, si pensi che nel territorio statunitense la $\mathrm{E}_{\mathrm{A}}$ è intorno a $24^{\circ}$ a New York $\left(41^{\circ} \mathrm{N}\right)$, mentre è di soli $10^{\circ}$ ad Astoria (sul Pacifico, $46^{\circ} \mathrm{N}$ ), oppure che nel blocco euroasiatico la $\mathrm{E}_{\mathrm{A}}$ è di circa $33^{\circ}$ a Vladivostok $\left(43^{\circ} \mathrm{N}\right.$, sul Pacifico), quando è di soli $14^{\circ}$ a La Rochelle $\left(46^{\circ} \mathrm{N}\right.$, nel litorale atlantico della Francia). Forti differenze nei caratteri climatici sono poi generate dall'eventuale presenza di catene montuose con sviluppo parallelo alle coste, per l'ostacolo che esse pongono all'arrivo delle masse d'aria oceaniche nelle zone sottovento; è il caso, ad esempio, delle Montagne Rocciose, delle Alpi Scandinave e anche - ad una dimensione geografica ben minore - dell'Appennino ligure, che separa nettamente il clima subcontinentale della Padania occidentale da quello prettamente mediterraneo dei litorali della Liguria.

Fondamentale è inoltre ricordare che l'influenza del mare sul clima non si limita al contenimento delle escursioni - oltre a quella annua, tende a diminuire anche la diurna - ma determina un'evidente modificazione del regime intermensile, dato che l'effetto di volano termico della massa d'acqua induce un maggiore ritardo nella risposta delle temperature rispetto alle culminazioni astronomiche. Questo si traduce nella tendenza allo spostamento dell'acme del freddo da gennaio a febbraio e di quello del caldo da luglio ad agosto (come ovvio, per l'emisfero boreale); tuttavia, l'aspetto più evidente del suddetto ritardo è però la marcata differenza che si crea fra le stagioni intermedie, con l'autunno che diviene decisamente più caldo della primavera (Pinna 2017).

Se è logico ritenere che tutte le considerazioni finora fatte dovrebbero rientrare nel bagaglio di conoscenze di chiunque si voglia avvicinare a studi di climatologia, di certo assai meno noto è il fatto che il livello di continentalità (o di oceanicità) dipende in modo considerevole pure dall'altitudine. L'obiettivo del presente lavoro è in effetti proprio quello di presentare alcune semplici verifiche, atte a dimostrare come, all'aumentare della quota, corrispondano dei caratteri termici sempre più spiccatamente marittimi.

Nella trattazione che segue, al fine di evidenziare i vari aspetti di cui detto, viene fatto riferimento all'uso dei seguenti parametri:

- $\mathrm{E}_{\mathrm{A}}=$ escursione annua;

- $\mathrm{E}_{\mathrm{D}}=$ escursione diurna media annua;

- $\left(\mathrm{T}_{1}-\mathrm{T}_{2}\right)=$ differenza fra le medie di gennaio e di febbraio;

- $\left(\mathrm{T}_{8}-\mathrm{T}_{7}\right)=$ differenza fra le medie di agosto e di luglio;

- $\left(\mathrm{T}_{10}-\mathrm{T}_{4}\right)=$ differenza fra le medie di ottobre ed aprile;

- $\left(\mathrm{T}_{\mathrm{IV}}-\mathrm{T}_{\mathrm{II}}\right)=$ differenza fra le medie dell'autunno e della primavera.

\section{Correlazioni fra altitudine e parametri di marittimità}

Sono state calcolate, per tre insiemi di stazioni, le regressioni lineari fra i valori della quota sul mare e dei parametri sopra citati (Pinna 2018); è importante precisare che i gruppi di stazioni sui quali sono state effettuate le analisi rispettano - almeno in linea di massima - le due condizioni essenziali per ottenere dei risultati attendibili e cioè essere riferiti ad aree geografiche con limitate variazioni latitudinali e non influenzate direttamente dalla vicinanza con i mari. Le tre aree studiate sono: a) un'ampia parte dell'Italia settentrionale; b) l'Alto Adige; c) l'intero territorio svizzero.

Il primo gruppo è costituito da 32 stazioni comprese nel grande database NOAA-GSOD ${ }^{1}$. Sono state escluse quelle della Liguria, in quanto poste a sud dell'arco appenninico e perciò con caratteristiche climatiche del tutto diverse dalle padane; per il settore orientale, è sembrato logico non valutare le località situate a meno di 50 $\mathrm{km}$ dalle coste dell'Adriatico, così da evitare gli effetti locali di tale bacino marino. Le elaborazioni sono state fatte sui dati del trentennio 1988-2017.

E parso opportuno riservare una trattazione separata all'Alto Adige, per il fatto che, trattandosi di

\footnotetext{
${ }^{1}$ National Oceanic and Atmospheric Administration, Global Surface Summary of the Day: https://data.noaa.gov/dataset/dataset/globalsurface-summary-of-the-day-gsod e https://www1.ncdc.noaa.gov/pub/ data/gsod/
} 
regione interamente priva di ampie zone pianeggianti, in essa dominano gli aspetti dovuti al contrasto fra rilievi e fondo valle. Si tratta di 30 stazioni i cui dati sono resi disponibili sul sito web dell'ufficio meteo della Provincia di Bolzano ${ }^{2}$; anche in questo caso, i calcoli sono stati eseguiti sulle medie del periodo 1988-2017.

A riguardo della Svizzera, l'ufficio federale di climatologia e meteorologia (Meteosvizzera) ${ }^{3}$ mette a disposizione sul proprio sito web i valori medi trentennali 19812010 di varie grandezze; per le temperature le stazioni censite sono 91, tutte prese complessivamente in carico nel calcolo delle regressioni in oggetto. In effetti, essendo quello elvetico un territorio di non grande estensione, nel quale non si realizzano considerevoli differenze di latitudine e di distanza dal mare, non è sembrato necessario effettuare alcuna selezione tra le località e neppure utile suddividerle in sottoinsiemi.

La tabella 1 è relativa alla ripartizione delle stazioni in sei classi di altimetria, mentre la tabella 2 contiene $\mathrm{i}$ risultati delle elaborazioni, con l'indicazione dei coefficienti di determinazione, utili a valutare i livelli di significatività delle correlazioni ottenute.

Tabella 1. Ripartizione delle stazioni utilizzate, secondo classi di altimetria.

\begin{tabular}{lccc}
\hline $\mathrm{h}(\mathrm{m})$ & Nord Italia & Alto Adige & Svizzera \\
\hline $0-250$ & 16 & 2 & 1 \\
$251-500$ & 2 & 3 & 28 \\
$501-1000$ & 5 & 8 & 20 \\
$1001-1500$ & 4 & 14 & 18 \\
$1501-2000$ & 1 & 3 & 17 \\
$>2000$ & 4 & 0 & 8 \\
\hline
\end{tabular}

La prima considerazione da fare è che, fra le 18 equazioni ricavate, ben 15 hanno degli $\mathrm{R}^{2}$ sufficienti a conferire un grado di confidenza del $99,9 \%$, con le tre rimanenti per le quali non si scende comunque al di sotto del $95 \%$. Questo dimostra che l'analisi di tutti i sei indicatori ha confermato un incremento dei caratteri di oceanicità, procedendo dalla pianura verso l'alto. Ovviamente, come evidenziato dalle notevoli differenze nei valori dei coefficienti di determinazione, alcune correlazioni sono risultate decisamente strette, altre meno; da rilevare che nei tre gruppi gli $\mathrm{R}^{2}$ corrispondenti al confronto fra le stagioni intermedie sono sempre assai elevati.

\footnotetext{
${ }^{2}$ http://meteo.provincia.bz.it/dati-storici.asp

${ }^{3}$ http://www.meteosvizzera.admin.ch/home/clima
}

Tabella 2. Regressioni ottenute per i vari indicatori considerati (h in $\mathrm{km}$ ).

\begin{tabular}{ll}
\hline \multicolumn{2}{c}{ Nord Italia } \\
\hline $\mathrm{E}_{\mathrm{A}}=-2,02 \cdot h+21,05$ & $\left(\mathrm{R}^{2}=0,81\right)$ \\
$\mathrm{E}_{\mathrm{D}}=-1,50 \cdot h+9,81$ & $\left(\mathrm{R}^{2}=0,44\right)$ \\
$\left(\mathrm{T}_{1}-\mathrm{T}_{2}\right)=0,77 \cdot h-1,91$ & $\left(\mathrm{R}^{2}=0,74\right)$ \\
$\left(\mathrm{T}_{8}-\mathrm{T}_{7}\right)=0,07 \cdot h-0,25$ & $\left(\mathrm{R}^{2}=0,13\right)$ \\
$\left(\mathrm{T}_{10}-\mathrm{T}_{4}\right)=0,80 \cdot h+1,44$ & $\left(\mathrm{R}^{2}=0,65\right)$ \\
$\left(\mathrm{T}_{\mathrm{IV}}-\mathrm{T}_{\mathrm{II}}\right)=0,64 \cdot h+0,76$ & $\left(\mathrm{R}^{2}=0,64\right)$ \\
\hline
\end{tabular}

\begin{tabular}{ll}
\hline \multicolumn{2}{c}{ Alto Adige } \\
\hline $\mathrm{E}_{\mathrm{A}}=-2,81 \cdot h+23,34$ & $\left(\mathrm{R}^{2}=0,63\right)$ \\
$\mathrm{E}_{\mathrm{D}}=-1,59 \cdot h+12,33$ & $\left(\mathrm{R}^{2}=0,27\right)$ \\
$\left(\mathrm{T}_{1}-\mathrm{T}_{2}\right)=1,47 \cdot h-3,27$ & $\left(\mathrm{R}^{2}=0,65\right)$ \\
$\left(\mathrm{T}_{8}-\mathrm{T}_{7}\right)=0,11 \cdot h-0,46$ & $\left(\mathrm{R}^{2}=0,20\right)$ \\
$\left(\mathrm{T}_{10}-\mathrm{T}_{4}\right)=2,04 \cdot h-1,15$ & $\left(\mathrm{R}^{2}=0,84\right)$ \\
$\left(\mathrm{T}_{\mathrm{IV}}-\mathrm{T}_{\mathrm{II}}\right)=1,82 \cdot h-1,68$ & $\left(\mathrm{R}^{2}=0,89\right)$ \\
& \\
\hline \multicolumn{1}{c}{ Svizzera } & $\left(\mathrm{R}^{2}=0,46\right)$ \\
$\mathrm{E}_{\mathrm{A}}=-1,64 \cdot h+19,45 \quad$ & $\left(\mathrm{R}^{2}=0,14\right)$ \\
$\mathrm{E}_{\mathrm{D}}=-1,01 \cdot h+9,62 \quad$ & $\left(\mathrm{R}^{2}=0,64\right)$ \\
$\left(\mathrm{T}_{1}-\mathrm{T}_{2}\right)=0,76 \cdot h-1,46$ & $\left(\mathrm{R}^{2}=0,55\right)$ \\
$\left(\mathrm{T}_{8}-\mathrm{T}_{7}\right)=0,24 \cdot h-0,78$ & $\left(\mathrm{R}^{2}=0,83\right)$ \\
$\left(\mathrm{T}_{10}-\mathrm{T}_{4}\right)=1,68 \cdot h+0,29$ & $\left(\mathrm{R}^{2}=0,83\right)$ \\
$\left(\mathrm{T}_{\mathrm{IV}}-\mathrm{T}_{\mathrm{II}}\right)=1,34 \cdot h-0,26$ &
\end{tabular}

Il campione del Nord Italia ha mostrato la relazione più forte per l'escursione annua (Fig.1), con una riduzione della stessa che si assesterebbe intorno ai $2^{\circ}$ ogni 1000 metri di quota. In pianura, ottobre è più caldo di aprile di circa $1,5^{\circ}$ in media, mentre nelle stazioni più elevate tale scarto arriva anche a superare i $4^{\circ}$.

Per l'Alto Adige, molto strette risultano le correlazioni relative ai parametri delle stagioni intermedie $\left(\mathrm{R}^{2}\right.$ pari a 0,84 e 0,89 ); in proposito, si è notato che le relative differenze termiche crescono molto con l'altitudine, passando da valori quasi sempre negativi nei fondivalle a $+2^{\circ}$ e oltre in montagna.

Rispetto ai dati dei due gruppi italiani, le equazioni delle escursioni della Svizzera hanno coefficienti di determinazione assai più bassi e presentano anche dei minori ratei di riduzione con l'altitudine; infatti per la $\mathrm{E}_{\mathrm{A}}$ si avrebbe un calo di circa $1,6^{\circ}$ ogni $\mathrm{km}$ di altezza. La forza delle altre regressioni è piuttosto alta, anche per il parametro $\left(\mathrm{T}_{8}-\mathrm{T}_{7}\right)$ che nei due precedenti esempi era invece caratterizzato da $\mathrm{R}^{2}$ assai minori. I livelli di confidenza decisamente maggiori riguardano le espressioni relative al confronto fra le stagioni intermedie (Fig. 2). 


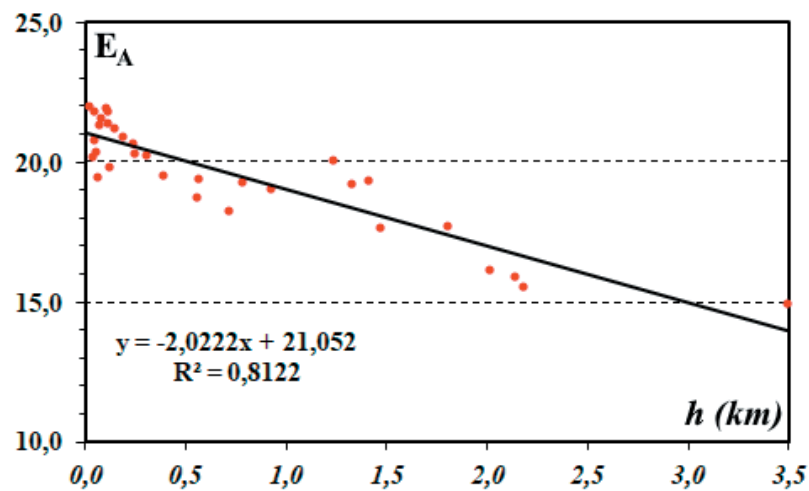

Figura 1. Correlazione fra altimetria ed escursione termica annua, per le 32 stazioni dell'Italia settentrionale. Fonte: Pinna S. 2018.

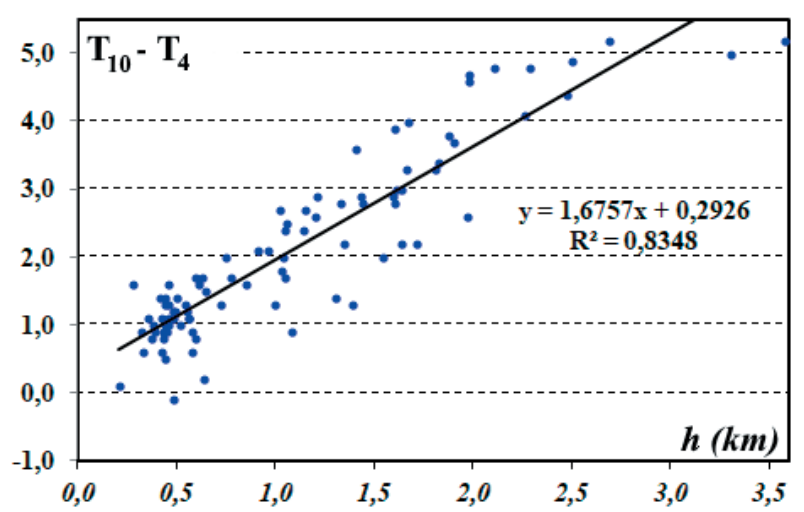

Figura 2. Correlazione, per le stazioni della Svizzera, fra altimetria e differenza termica ottobre-aprile. Fonte: Pinna S. 2018.

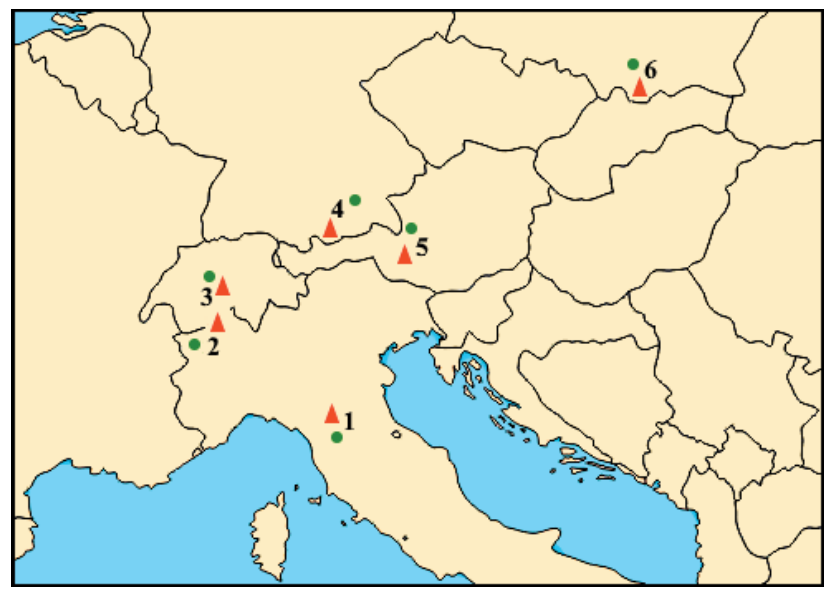

Figura 3. La localizzazione delle coppie di stazioni di cui nel testo. 1 = Monte Cimone (2173 m) e Pistoia (65); 2 = Plateau Rosa (3488) e Aosta (551); 3 = Jungfraujoch (3580) e Interlaken (557); 4 = Zugspitze (2960) e Monaco di Baviera (521); 5 = Sonnblick (3109) e Salisburgo (437); 6 = Kasprowy Wierch (1987) e Cracovia (217).

\section{Alcuni esempi di regime termico in alta montagna}

L'interpretazione derivante dai risultati delle analisi statistiche di cui si è appena riferito viene pienamente confermata dallo studio dei regimi termici delle stazioni d'alta quota, che risultano avere appunto dei caratteri corrispondenti a quelli di tipiche località costiere. In questo paragrafo ne saranno esaminati alcuni esempi, proponendoli sempre in confronto con i dati di stazioni di pianura situate nella stessa regione geografica di appartenenza di quelle di montagna (Fig. 3).

Tutti i casi esaminati attestano come in alta quota il clima abbia parametri di oceanicità decisamente superiori a quelli delle vicine pianure (Tab. 3). Soltanto Plateau Rosa, nonostante la sua altitudine, ha caratteristiche non troppo spinte in tal senso; in ogni caso, comunque ben discoste da quelle di Aosta.

Jungfraujoch, Zugspitze e Sonnblick hanno una $\mathrm{E}_{\mathrm{A}}$ inferiore ai $15^{\circ}$ e una temperatura media del mese di ottobre maggiore di circa $5^{\circ}$ a quella d'aprile; per avere un dato di riferimento, si pensi che mediando fra tutte le stazioni marine di Sardegna e Sicilia, presenti nel database NOAA-GSOD, i valori di tali parametri risultano di $15,3^{\circ}$ e $5,3^{\circ}$.

Tabella 3. Parametri di continentalità/oceanicità per sei stazioni d'alta montagna, ognuna raffrontata con un'altra di pianura della stessa area geografica.

\begin{tabular}{lrrrr}
\hline & Pl. Rosa & Aosta & Jungfraujoch & Interlaken \\
\hline quota & 3488 & 551 & 3580 & 557 \\
$\mathrm{E}_{\mathrm{A}}$ & 15,0 & 18,8 & 13,7 & 18,7 \\
$\left(\mathrm{~T}_{1}-\mathrm{T}_{2}\right)$ & 0,4 & $-1,8$ & 0,8 & $-1,2$ \\
$\left(\mathrm{~T}_{8}-\mathrm{T}_{7}\right)$ & 0,2 & 0,0 & 0,0 & $-0,7$ \\
$\left(\mathrm{~T}_{10}-\mathrm{T}_{4}\right)$ & 4,3 & 2,0 & 5,2 & 0,9 \\
$\left(\mathrm{~T}_{\mathrm{IV}}-\mathrm{T}_{\mathrm{II}}\right)$ & 3,1 & 1,2 & 3,6 & 0,3 \\
\hline & Zugspitze & Monaco B. & Sonnblick & Salisburgo \\
\hline $\mathrm{quota}$ & 2960 & 521 & 3109 & 437 \\
$\mathrm{E}_{\mathrm{A}}$ & 13,7 & 19,2 & 14,5 & 19,3 \\
$\left(\mathrm{~T}_{1}-\mathrm{T}_{2}\right)$ & 0,5 & $-1,2$ & 0,5 & $-1,7$ \\
$\left(\mathrm{~T}_{8}-\mathrm{T}_{7}\right)$ & 0,4 & $-0,7$ & 0,2 & $-0,1$ \\
$\left(\mathrm{~T}_{10}-\mathrm{T}_{4}\right)$ & 5,1 & 0,7 & 4,6 & 1,1 \\
$\left(\mathrm{~T}_{\mathrm{IV}}-\mathrm{T}_{\mathrm{II}}\right)$ & 3,5 & 0,7 & 3,3 & 0,3 \\
\hline & Kasprowy & Cracovia & M. Cimone & Pistoia \\
\hline $\mathrm{quota}$ & 1987 & 217 & 2173 & 65 \\
$\mathrm{E}_{\mathrm{A}}$ & 15,3 & 21,5 & 15,6 & 18,6 \\
$\left(\mathrm{~T}_{1}-\mathrm{T}_{2}\right)$ & 0,0 & $-1,9$ & 0,5 & $-1,5$ \\
$\left(\mathrm{~T}_{8}-\mathrm{T}_{7}\right)$ & 0,1 & $-0,4$ & 0,1 & $-0,4$ \\
$\left(\mathrm{~T}_{10}-\mathrm{T}_{4}\right)$ & 3,9 & 0,6 & 4,4 & 2,1 \\
$\left(\mathrm{~T}_{\mathrm{IV}}-\mathrm{T}_{\text {II }}\right)$ & 2,9 & 0,5 & 3,1 & 2,0 \\
\hline & & & &
\end{tabular}


La figura 4 mostra i regimi termici delle due località bavaresi, Zugspitze e Monaco di Baviera, in base a valori che sono stati corretti al livello del mare - secondo il teorico gradiente altimetrico di $6,5^{\circ} / \mathrm{km}$ utilizzato nel modello dell'atmosfera standard - onde permettere di apprezzare meglio le diversità climatiche negli aspetti di continentalità. Rispetto a Monaco infatti, la località montana, oltre a presentare un'escursione complessiva ben minore, ha un andamento che, se abbastanza simile nel periodo aprile-luglio, ne diverge poi chiaramente, denotando così una stagione autunnale tipicamente marittima.

Assai interessanti anche i due rimanenti esempi, sia per la minore quota delle stazioni montane, sia per la loro collocazione geografica. Kasprowy (Polonia), situata sui Monti Tatra e pertanto ad una distanza di quasi 900 $\mathrm{km}$ dal Mare del Nord, ha dei valori nettamente differenti da quelli di Cracovia; meno di $1800 \mathrm{~m}$ di dislivello sono pertanto sufficienti per passare da un clima continentale ad uno di tipologia marittima. Il raffronto infine tra il M. Cimone e Pistoia dimostra come i fenomeni in oggetto si possano ben riconoscere pure nella catena appenninica.

\section{Una valutazione del grado di oceanicità mediante un indicatore sintetico}

Onde consentire una valutazione sintetica degli aspetti climatologici in oggetto, è normale il ricorso al calcolo di opportuni indicatori; per quanto riguarda l'oceanicità, quello forse più utilizzato è l'indice di Kerner (KOI), la cui espressione è la seguente:

$$
\mathrm{KOI}=100 \cdot\left(\mathrm{T}_{10}-\mathrm{T}_{4}\right) / \mathrm{E}_{\mathrm{A}}
$$

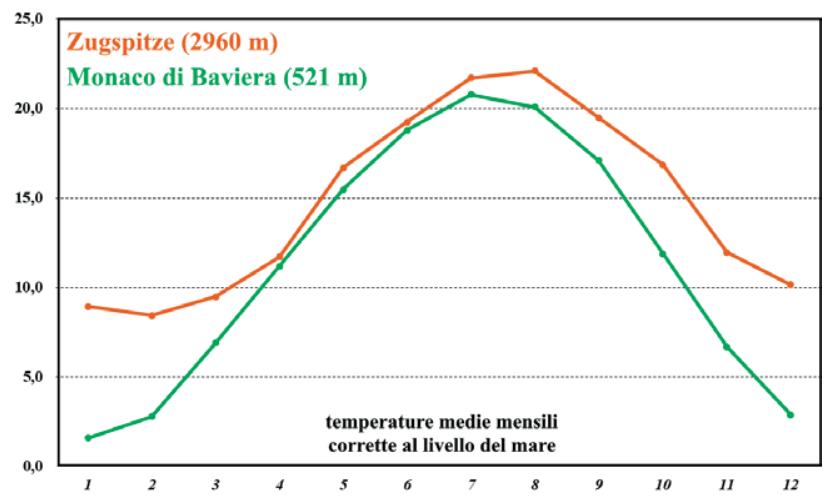

Figura 4. Il regime termico di Zugspitze e di Monaco, secondo temperature riportate al livello del mare. Sono evidenti le forti differenze nei caratteri di continentalità.
In questa sede, ho ritenuto opportuno proporre un nuovo possibile indicatore complessivo, sempre basato sui dati delle temperature medie mensili, ma che considerasse non più il solo parametro $\left(\mathrm{T}_{10}-\mathrm{T}_{4}\right)$, bensì anche quelli relativi ai mesi di acme del freddo e del caldo.

In ogni formula di questo genere, come ovvio, deve rientrare pure la $\mathrm{E}_{\mathrm{A}}$, in merito alla quale bisogna sempre ricordare che essa dipende sia dall'influsso marino, sia dalla latitudine $(\varphi)$. Pertanto, quando si vuole cercare di valutarla soltanto in funzione del suo ammontare dovuto al primo di tali fattori, è necessario apportare delle correzioni.

Per affrontare il problema, sono partito dall'espressione che lega $\varphi$ all'escursione annua, ricavata da Pearce e Smith (1990), mediante una correlazione su 142 stazioni di Europa, Africa e Sud America:

$$
\mathrm{E}=0,4 \cdot \varphi
$$

Vista la dispersione dei dati segnalata dagli Autori, essa va evidentemente ritenuta come una stima solo di primo ordine, ma ritengo che possa però servire per calcolare un teorico valore mediano $\left(\mathrm{E}_{\mathrm{t}}\right)$ dell'escursione annua, cui rapportare la reale $\mathrm{E}_{\mathrm{A}}$; in pratica, se $\mathrm{E}_{\mathrm{A}}>\mathrm{E}_{\mathrm{t}}$ si ipotizza una condizione di continentalità, al contrario una di marittimità.

Sulla scorta delle varie osservazioni ora fatte, l'indicatore complessivo di marittimità (COI) che suggerisco, utilizzabile per le regioni extratropicali, viene calcolato come somma algebrica dei seguenti quattro termini:

$$
\begin{aligned}
& 2 \cdot\left(\mathrm{T}_{1}-\mathrm{T}_{2}\right) \\
& \text { - } 2 \cdot\left(\mathrm{T}_{8}-\mathrm{T}_{7}\right) \\
& \text { - } 1,5 \cdot\left(\mathrm{T}_{10}-\mathrm{T}_{4}\right) \\
& \text { - } 1,5 \cdot\left(\mathrm{E}_{\mathrm{t}}-\mathrm{E}_{\mathrm{A}}\right)
\end{aligned}
$$

I coefficienti numerici assegnati ai singoli termini derivano da numerose verifiche empiriche effettuate, ma è ovvio che potrebbero essere ricalibrati, se future applicazioni del metodo dovessero far emergere dei problemi in certe interpretazioni climatologiche.

Valori negativi del COI denotano una continentalità, positivi una marittimità. Due stazioni ben note in bibliografia per il loro clima estremamente oceanico, Torshavn (nell'arcipelago delle Fær Øer) e l'isola Macquarie (a sud della Nuova Zelanda), ottengono rispettivamente valori di 29 e 27; ne deriva quindi che questi possano grosso modo rappresentare i livelli massimi raggiungibili. All'opposto, cioè in direzione della continentalità, dati inferiori a -10 ne mostrano una condizione già abbastanza spinta; Budapest, ad esempio, è intorno a -11 e Mosca a -16. Spostandosi all'interno dei conti- 
nenti, si può comunque arrivare a valori ben più bassi, in virtù di escursioni annue fortissime; un caso quasi limite dovrebbe essere rappresentato da Jakutsk, in Siberia ad una longitudine di $129^{\circ}$ Est, ove la $E_{\mathrm{A}}$ è di quasi $60^{\circ} \mathrm{e}$ l'indice COI scende addirittura a -74 .

Per l'Italia, si osserva il passaggio da valori negativi nella Padania e nei fondivalle alpini a dati di circa 10 nelle aree costiere meridionali; fra le stazioni rientranti nel database NOAA-GSOD, Bolzano ha il minimo (-9), mentre il COI supera 12 nelle isole del Canale di Sicilia.

Rispetto a quello di Kerner, l'indicatore ora presentato sembra in grado di distinguere meglio alcune situazioni. Ad esempio, si calcola un KOI intorno a 12 sia per Modena, sia per Trondheim, città costiera della Norvegia; il COI è invece circa di -3 per la prima e di 12 per la seconda, indicando quindi in modo più adeguato i diversi effetti marini sui caratteri climatici delle due città.

La tabella 4 riporta i valori dell'indice di Kerner e del COI per le coppie di stazioni già citate nella precedente tabella 3; si constata così sinteticamente che nelle vette alpine il clima può avere caratteri di marittimità talmente spinti, da dar luogo a entità degli indici abbastanza vicine a quelle massime conosciute al livello del mare.

Tabella 4. Valori dell'indice di Kerner (KOI) e di quello elaborato in questa sede (COI), per le stazioni di cui alla tabella precedente.

\begin{tabular}{lrrrr}
\hline & Pl. Rosa & Aosta & Jungfraujoch & Interlaken \\
\hline quota & 3488 & 551 & 3580 & 557 \\
KOI & 28,6 & 10,6 & 38,0 & 4,8 \\
COI & 12,9 & $-1,3$ & 16,8 & $-2,5$
\end{tabular}

\begin{tabular}{lrrrr}
\hline & Zugspitze & Monaco B. & Sonnblich & Salisburgo \\
\hline quota & 2960 & 521 & 3109 & 437 \\
KOI & 37,5 & 3,6 & 31,4 & 5,6 \\
COI & 17,4 & $-2,7$ & 14,6 & $-2,2$
\end{tabular}

\begin{tabular}{lrrrr}
\hline & Kasprowy & Cracovia & M. Cimone & Pistoia \\
\hline quota & 1987 & 217 & 2173 & 65 \\
KOI & 25,5 & 2,8 & 28,6 & 11,3 \\
COI & 12,6 & $-6,0$ & 11,1 & $-2,2$ \\
\hline
\end{tabular}

\section{Brevi considerazioni conclusive}

Le verifiche condotte hanno complessivamente confermato, per territori non direttamente esposti all'influenza marina, il forte impatto dell'altitudine sui caratteri di marittimità, nel verso di una marcata crescita di quest'ultima con l'aumentare della quota sul livello del mare. In pratica, passando dalle stazioni più basse a quelle di maggiore elevazione, si è osservato lo stesso tipo di modificazione generale del regime termico, che si realizza quando si procede da un'area interna verso la costa.

Rolland (2003) ritiene che l'altitudine incida direttamente sulla $\mathrm{E}_{\mathrm{A}}$ in ragione sia del ridotto assorbimento della radiazione ad onda lunga in uscita (a causa della rarefazione dell'aria), sia della maggiore esposizione ai venti degli strati superiori dell'atmosfera. Questo secondo fattore - se consideriamo la questione della marittimità nel suo complesso - sembra invero essere particolarmente rilevante, quando si pensi soprattutto all'andamento delle altre variabili studiate in questo lavoro e cioè a quelle legate agli spostamenti in avanti delle manifestazioni termiche, rispetto ai momenti dei solstizi.

Tabella 5. Parametri climatici e indice COI per la stazione di Milano Linate, a livello del suolo e alle quote approssimative indicate.

\begin{tabular}{lrrrrr}
\hline & $\mathrm{E}_{\mathrm{A}}$ & $\left(\mathrm{T}_{1}-\mathrm{T}_{2}\right)$ & $\left(\mathrm{T}_{8}-\mathrm{T}_{7}\right)$ & $\left(\mathrm{T}_{10}-\mathrm{T}_{4}\right)$ & \multicolumn{1}{c}{$\mathrm{COI}$} \\
\hline al suolo & 21,5 & $-2,2$ & $-0,4$ & 1,4 & $-8,0$ \\
$\sim 1500 \mathrm{~m}$ & 16,3 & 0,5 & $-0,3$ & 2,8 & 7,5 \\
$\sim 3300 \mathrm{~m}$ & 13,9 & 0,8 & 0,0 & 4,8 & 15,2 \\
\hline
\end{tabular}

Si può in definitiva ritenere che, muovendosi verso l'alto lungo i versanti, si incontrino condizioni climatiche nelle quali tendono a divenire predominanti gli apporti oceanici, per l'azione della circolazione generale in quota. Un quadro confermato anche dalle misure condotte ad altitudini diverse con i radiosondaggi, come nel caso della stazione di Milano Linate (Tab. 5); si può infatti constatare che, ad una altezza di circa $3300 \mathrm{~m}$, i dati termici sono del tutto simili a quelli delle località montane prima citate.

\section{Fonte dei dati}

National Oceanic and Atmospheric Administration, Global Surface Summary of the Day, https://www1.ncdc. noaa.gov/pub/data/gsod/

Provincia di Bolzano, http://meteo.provincia.bz.it/download-dati.asp

Meteosvizzera, http://www.meteosvizzera.admin.ch/ home/clima

World Weather Information Service. Official Forecasts, http://worldweather.wmo.int/en/home.html

Rimfrost, http://www.rimfrost.no/

Climate-data.org, https://it.climate-data.org/ 


\section{Riferimenti bibliografici}

Barry, R. G. (2008). Mountain Weather \& Climate. Cambridge, Cambridge University Press.

Geerts, B. (2002). Empirical estimation of the annual range of monthly-mean temperature, Theoretical and Applied Climatology, 73, 107-32.

Pearce, E. A., Smith, C. G. (1990). The World Weather Guide. Hutchinson.

Pinna, M. (1991). Contributo alla conoscenza del grado di continentalità del clima dell'Italia. In Palagiano, C., Paratore, E. (a cura di), Studi in onore di Osvaldo Baldacci. Bologna, Pàtron, 5-17.

Pinna, S. (2017). Lineamenti di Climatologia. Roma, Aracne.

Pinna, S. (2018). Correlazioni fra altitudine e indicatori della continentalità. Alcune applicazioni per l'Italia settentrionale e per la Svizzera. Rivista di Meteorologia Aeronautica, 4, 5-12.

Rolland, C. (2003). Spatial and Seasonal Variations of Air Temperature Lapse Rates in Alpine Regions. Journal of Climate, 16, 1032-1046.

Stonevicius, E., Stankunavicius, G., Rimkus, E. (2018). Continentality and Oceanity in the mid and high latitudes of the Northern Hemisphere and their links to atmospheric circulation. Advances in Meteorology, Article ID 5746191. https://doi.org/10.1155/2018/5746191 